\title{
A STUDY ON DYNAMIC ANALYSIS OF A DAM INTAKE TOWER AND FOUNDATION
}

\author{
Ravikumara H $\mathbf{S}^{\mathbf{1}}$ \\ ${ }^{I}$ Research Scholar, Dept of Civil Engineering, National Institute of Technology Karnataka, Surathkal, Karnataka, \\ India
}

\begin{abstract}
The main objective of this study is to investigate the dynamic behavior of circular cylindrical intake towers. Intake towers are typically tall and hollow structures made of reinforced concrete and they form entrance to reservoir outlet works. The circular intake towers are structurally more efficient, providing economic savings, particularly in high-head projects. The effect of depth of submergence, slenderness ratio and wall thickness is studied. The hydrodynamic effects are accounted for using added mass concept. Also, the soil-structure-interaction analysis is carried out to study its effect on the dynamic behavior of the intake towers. The computer software package, SAP2000, is used for developing finite element models of intake tower and soil using shell elements and solids respectively. Modal and Response Spectrum analyses are carried out to determine the dynamic characteristics and response of the tower to earthquake excitation for various geometrical parameters and submergence conditions.
\end{abstract}

Keywords: Intake Towers, Dynamic analysis, Hydrodynamic effects, soil-structure interaction, SAP2000 $* * *$

\section{INTRODUCTION}

Outlet works of intake towers typically consist of an intake structure and a conduit or tunnel, in addition to other features necessary for operation, such as control gates, approach and discharge channels, and energy dissipaters [1]. There might be multiple openings in an intake structure to provide for the valves and gates that help control the flow of water and thus regulating water supply. The mechanism for controlling the water flow is generally housed within the intake tower.Intake towers can be separated into two categories: free-standing (vertical structure, typically with a service bridge at the top) and inclined (structure supported against the abutment).

This paper focuses on intake towers which are free-standing, as they represents the most common structural configuration.

\subsection{Problem Description}

Generally the only means of lowering the reservoir level and minimizing the extent of damage during a catastrophic failure such as an earthquake is the outlet works in various dams. This is very important as the controlled release of water can prevent additional damages as it reduces the hydrostatic loads at the outlet.

Therefore, it is extremely important to understand the dynamic behavior of a dam intake tower. However, the presence of water surrounding and inside the tower and the axisymmetric geometry makes the structure quite complex. Here the 3-dimensional model of the structure has been made for more accurate results and also to incorporate the effects of the water-structure-interaction. The effect of soil at the base of the tower which has significant effect on its dynamic responses and its stiffness is also taken into consideration for analysis and the results have compared with the results of a fixed base tower conditions.

\subsection{Objective}

Different parameters that considerably affect the behaviour of intake towers during the event of an earthquake such as Depth of submergence $\left(\mathrm{H}_{\delta} \mathrm{H}\right)$, The ratio Outside radius $\left(\mathrm{r}_{\mathrm{o}}\right)$ to height $(H)$ of tower, Wall thickness $(t)$, Effect of soilstructure interaction (SSI) have been considered and the dynamic analysis of dam intake tower and foundation have been carried out using SAP 2000.

\subsection{Modelling Of Intake Tower}

A simplified model of a uniform circular intake tower is considered for the analysis.

Using the concept of hydrodynamic added masses (Goyal and Chopra 1989), hydrodynamic effects were included in the analysis. The effect of soil at the base was modelled using block elements with a medium stiffness to provide necessary elasticity.

A circular tower of height $\mathrm{H}=61 \mathrm{~m}$ is considered for analysis as shown in Figure 1. A finite element model of shell elements is used in this study. The depth of surrounding and inside water is taken at two different levels i.e. $\mathrm{H}_{\mathrm{o}} / \mathrm{H}=0.5$ and $\mathrm{H}_{\mathrm{o}} / \mathrm{H}=1$, where $\mathrm{H}_{\mathrm{o}}$ is the level of water both inside and outside. Masses for these water levels are calculated according to the concept of added mass and applied to the tower at various nodes as lumped mass. The wall thickness $(\mathrm{t})$ and outer radius $\left(\mathrm{r}_{\mathrm{o}}\right)$ are varied as well to study the effects 
of these parameters on the dynamic properties of the tower. Soil is modelled as a block element as shown in Figure 7 and its effect on the dynamic behaviour is studied.

Modal and Response Spectrum analyses are carried in SAP2000 to obtain the dynamic properties and responses of the tower structure.

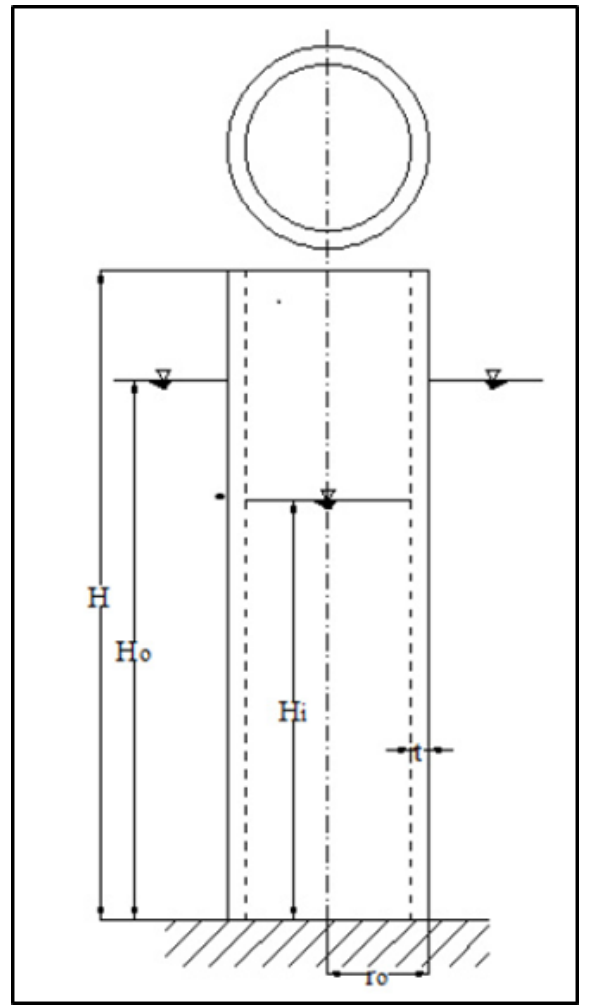

Fig 1 A typical intake tower

A 3-D shell model of a circular intake tower studied is shown in Figure 2.

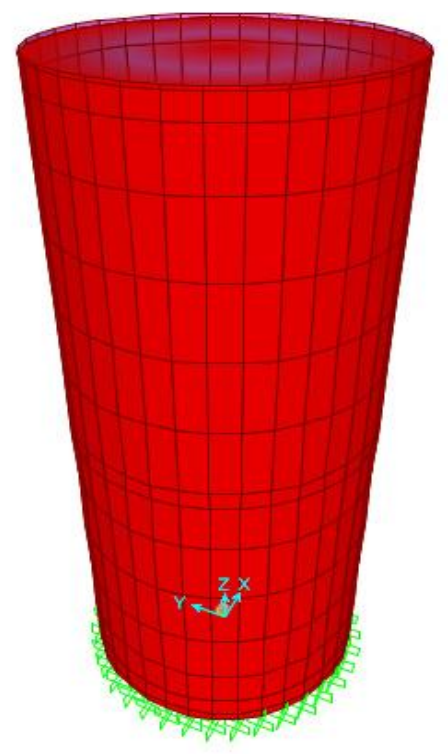

Fig 2 Circular Intake tower model

\section{CALCULATION OF HYDRODYNAMIC MASSES}

The effect of fluid-structure interaction on the tower has been considered which was obtained from the hydrodynamic mass of inside and outside water. The water is taken as an incompressible fluid and the mass of an intake tower $\mathrm{m}_{\mathrm{s}}(\mathrm{z})$ having an arbitrary cross-section with two axes of symmetry is replaced by the virtual mass $M_{\mathrm{s}}(\mathrm{z})$ as:

$$
\mathrm{M}_{\mathrm{s}}(\mathrm{z})=\mathrm{m}_{\mathrm{s}}(\mathrm{z})+\mathrm{m}_{\mathrm{a}}^{\mathrm{o}}(\mathrm{z})+\mathrm{m}_{\mathrm{a}}^{\mathrm{i}}(\mathrm{z})
$$

Here, the added hydrodynamic masses $\mathrm{m}_{\mathrm{a}}^{\mathrm{o}}(\mathrm{z})$ represents the effect outside water and $\mathrm{m}_{\mathrm{a}}^{\mathrm{i}}(\mathrm{z})$ represents the effect of inside water, on the dynamic response of the tower.

The graphs shown in Figure. 3 and Figure. 4 have been used to calculate the outside and inside hydrodynamic masses for a circular tower. The calculations were done using excel worksheets.

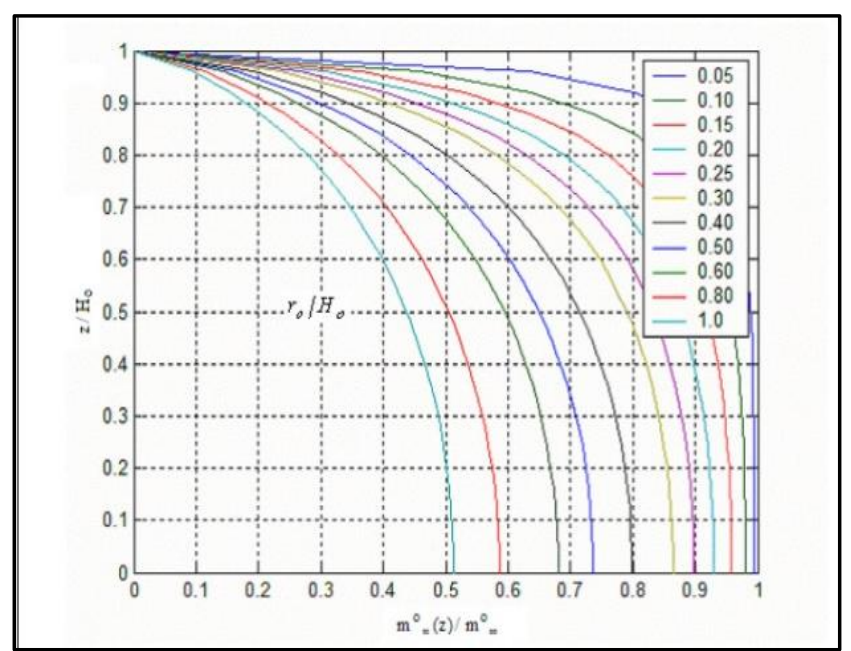

Fig 3 Normalised outside hydrodynamic added mass for circular cylinder towers

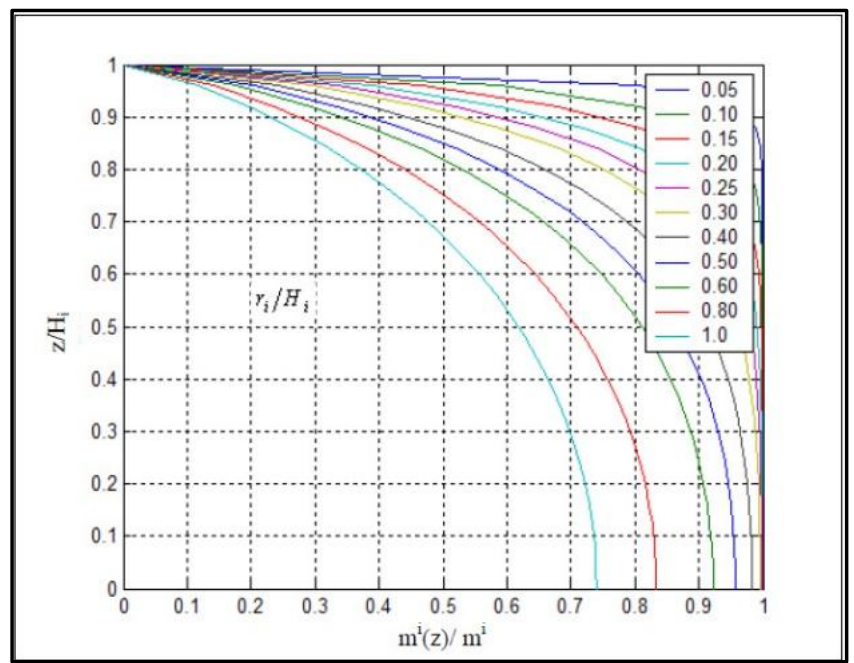

Fig 4 Normalised inside hydrodynamic mass for circular cylinderical towers 


\section{DYNAMIC ANALYSIS}

\subsection{Modal Analysis}

By considering Eigen vector method, modal analysis has been carried out. Eigenvector analysis determines the undamped free-vibration mode shapes and frequencies of the system. These natural modes provides an excellent insight into the behavior of the structure.

12 modes have been considered for the analysis. Modal analysis has been carried out for different levels of submergence, wall thickness and slenderness values and the resulting time periods are tabulated and compared to study the effects of these parameters.

\subsection{Effect of Submergence}

To study the effect of submergence on the dynamic properties, 3 conditions were chosen,

i.e. full water submergence $\mathrm{H}_{0} / \mathrm{H}=1$, half water $\mathrm{H}_{\mathrm{o}} / \mathrm{H}=0.5$ and no water condition.

It was observed that, time period is maximum when the tower is fully submerged and reduces as the depth of submergence decreases and comes to a minimum value at no water condition. The increase was very less for half submergence compared to the increase in the fully submerged condition. Therefore, for $\mathrm{H}_{\mathrm{o}} / \mathrm{H}=0.5$, the effect of water structure interaction need not be considered. The reason for maximum increase in period for fully submerged case may be due to maximum added mass of the water.

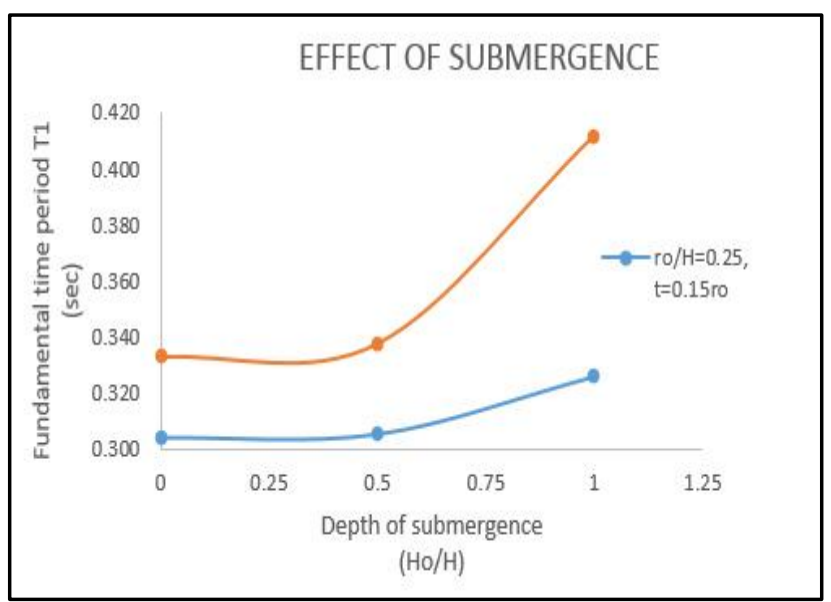

Fig 5 The variation of fundamental time period

The variation in fundamental time period $\mathrm{T} 1$ for different submergence conditions is shown in the graph 5 below:

\subsection{Effect of Slenderness}

To study the effect of slenderness ratio on the dynamic properties of the tower, three towers were selected. Slender tower $\mathrm{r}_{\mathrm{o}} / \mathrm{H}=0.05$, squatty tower having $\mathrm{r}_{\mathrm{o}} / \mathrm{H}=0.25$, and a third tower selected was in between squatty and slender with $\mathrm{r}_{\mathrm{o}} / \mathrm{H}=0.1$.
It has seen that there was nearly $112 \%$ increase in the time period for the fundamental mode, for the decrease in radius from $r_{o} / H=0.25$ to $r_{o} / H=0.1$. Similarly there is an increase of nearly $98 \%$ in the time period for the decrease in radius from $r_{0} / H=0.1$ to $r_{o} / H=0.05$. Therefore it can be observed that, as the slenderness of the tower increases there is an increase in the time periods of the fundamental modes with the radii of the towers of similar thicknesses and submergence. The variation in the fundamental mode with slenderness is shown in Figure 6.

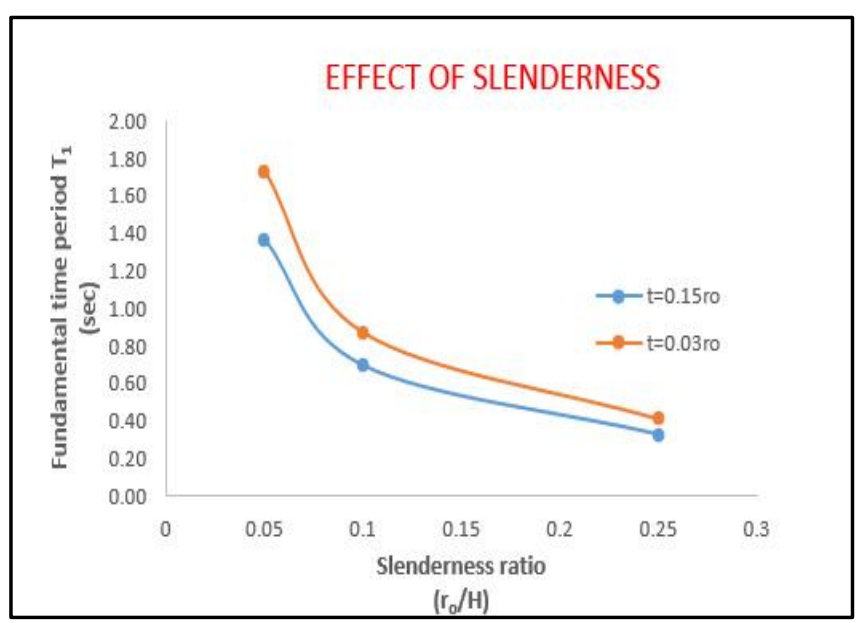

Fig 6 Variation of fundamental time period for different slenderness ratios

\subsection{Effect of Thickness}

Two different thicknesses $t=0.15 r_{o}$ and $t=0.03 r_{o}$ were selected for each radius to study the effect of thickness.

It was observed that there was a definite increase in the time period values as thickness decreases for the towers with same radius. The time period values were greater for towers with wall thickness $t=0.03 r_{0}$ than $t=0.15 r_{0}$, because the tower becomes more flexible as its thickness reduces. The graphical representation can be seen in Figure 6.

\subsection{Effect of Soil-Structure Interaction}

To observe the effects of SSI on the dynamic behaviour of the intake tower, a tower model with block elements to represent the soil below it was also developed in SAP2000 as shown in the figure 7 . The soil was taken as medium to hard condition. The radius of the tower taken was

$$
\mathrm{r}_{\mathrm{o}} / \mathrm{H}=0.25 \text { and thickness } \mathrm{t}=0.15 \mathrm{r}_{0} \text {. }
$$




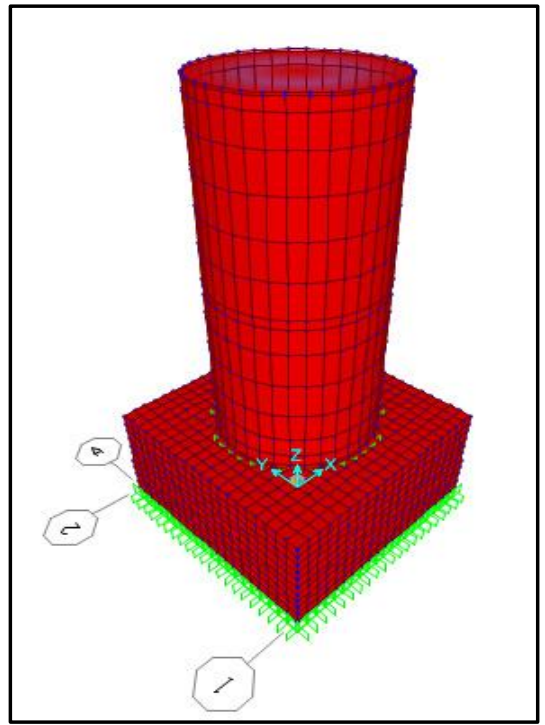

Fig 7 Intake tower with soil

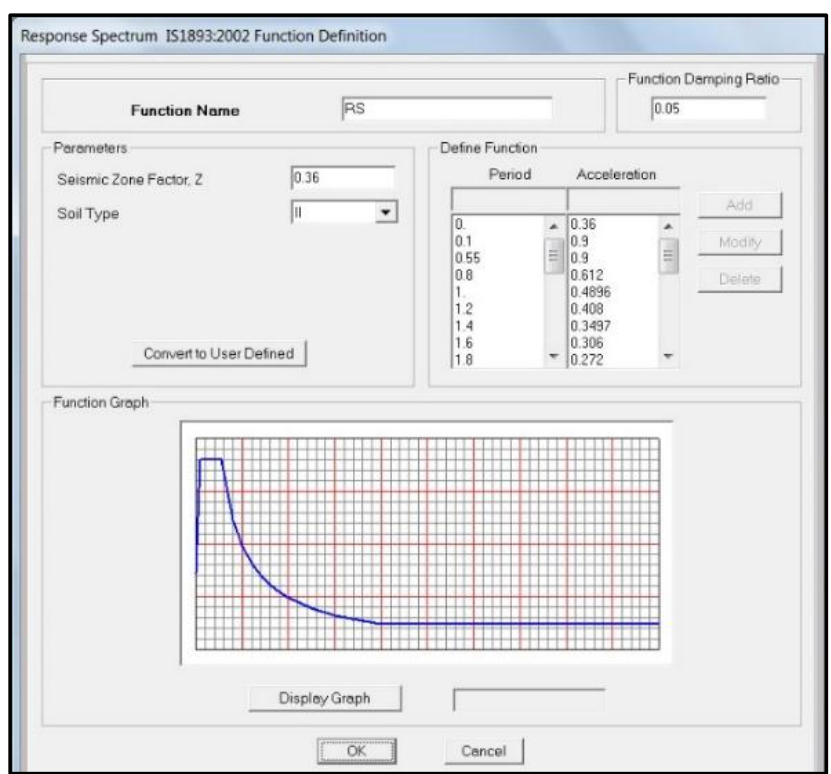

Fig 8 Details of response spectrum functions

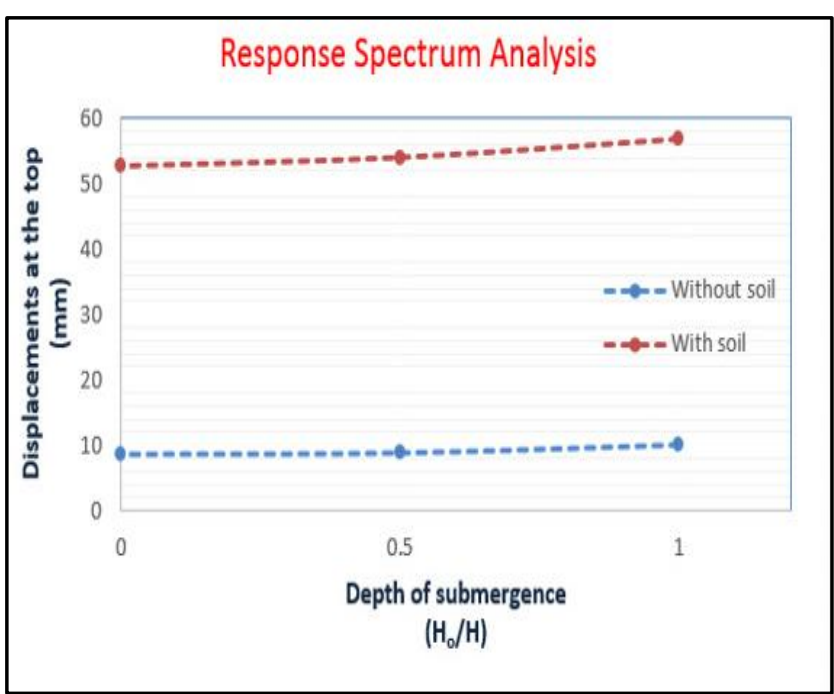

Fig 9 Displacements at the top of the tower for different submergence conditions

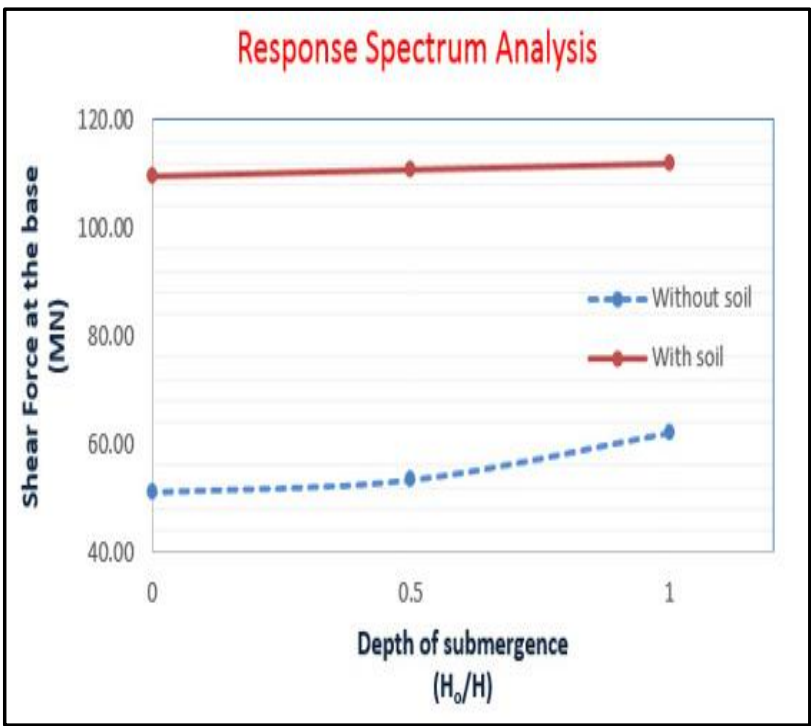

Fig 10 Shear forces at the base of the tower for different submergence conditions

Modal analysis was conducted for the soil model for different submergence conditions and compared the time periods with the results obtained for the same model with fixed base. The percentage increase in the time periods is shown in Table 1.

Table 1 Increase in the modal time periods with the SSI

\begin{tabular}{|l|l|l|l|}
\hline & \multicolumn{3}{|c|}{ increase in Time period } \\
\hline $\begin{array}{l}\text { Time } \\
\text { period }\end{array}$ & $\mathbf{H}_{\mathbf{O}} / \mathbf{H}=\mathbf{1}$ & $\begin{array}{l}\mathbf{H}_{\mathbf{O}} / \mathbf{H}= \\
\mathbf{0 . 5}\end{array}$ & $\begin{array}{l}\text { No } \\
\text { water }\end{array}$ \\
\hline $\mathbf{T}_{\mathbf{1}}$ & 213.30 & 214.84 & 215.02 \\
\hline $\mathbf{T}_{\mathbf{2}}$ & 213.73 & 212.13 & 210.49 \\
\hline $\mathbf{T}_{\mathbf{3}}$ & 43.76 & 39.29 & 37.30 \\
\hline $\mathbf{T}_{\mathbf{4}}$ & 22.20 & 25.55 & 27.03 \\
\hline $\mathbf{T}_{\mathbf{5}}$ & 21.44 & 25.02 & 23.81 \\
\hline $\mathbf{T}_{\mathbf{6}}$ & 21.51 & 23.27 & 24.12 \\
\hline $\mathbf{T}_{\mathbf{7}}$ & 20.67 & 23.13 & 22.82 \\
\hline $\mathbf{T}_{\mathbf{8}}$ & 45.41 & 51.53 & 51.76 \\
\hline $\mathbf{T}_{\mathbf{9}}$ & 36.26 & 36.39 & 37.53 \\
\hline $\mathbf{T}_{\mathbf{1 0}}$ & 66.33 & 66.44 & 66.39 \\
\hline $\mathbf{T}_{\mathbf{1 1}}$ & 59.07 & 65.77 & 66.25 \\
\hline $\mathbf{T}_{\mathbf{1 2}}$ & 35.02 & 34.09 & 32.79 \\
\hline
\end{tabular}

Hence it was seen that the effect of soil causes a significant increase in the fundamental time periods, therefore it is very important to consider the effect of soil for the dynamic analysis of the tower.

\section{RESPONSE SPECTRUM ANALYSIS}

Response Spectrum analysis was conducted on a circular tower model to observe its dynamic responses. Response spectrum Analysis was also conducted on the model shown in Figure 7 to study the effects of Soil Structure Interaction on its dynamic behaviour. A response reduction factor of 5 and importance factor 1.5 was used. 
To study the effects of SSI on the dynamic responses of the intake tower, base reactions and displacements at the top of the tower between fixed base and flexible base (with soil) for different submergence conditions. Obtained with RSA are shown in the graphs 9,10 and 11 below.

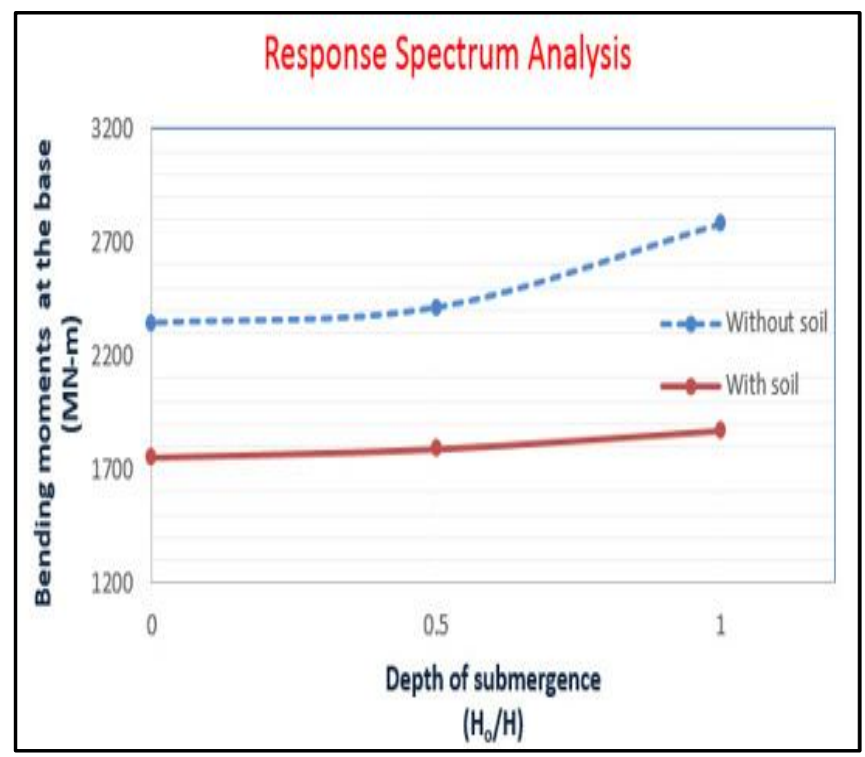

Fig 11 Bending moments at the base of the tower for different submergence conditions

It was seen that there is an increase in the displacements at the top as well as the shear force at the base when the effect of SSI is considered, due to the added mass of the soil. However, due to the increase in flexibility at the base, the bending moment tends to decrease in value.

\section{CONCLUSION}

On the basis of analysis and results, It has been concluded that, the presence of water inside and outside of the structure for a fully submerged tower increases the fundamental time period of the tower due to the added mass. The fundamental time period increases as the slenderness of the tower increases. This is due to the increase in flexibility of the tower.As the thickness of the tower decreased, the time periods also decreased due to the decrease in self weight of the tower.

There was a great increase in time periods when the soil was modelled at the base.Displacement at the top was much greater for the tower with base soil effect. Bending moment was lesser for soil model due to increased flexibility. Shear force at the base was increased for model with flexible base as the seismic weight due to the soil increases. The effect on fundamental time period increases with increased soil flexibility.

\section{REFERENCES}

[1]. U.S.Army Corps of Engineers. (2003). "Structural Design and Evaluation of Outlet Works", Engineer Manual, Washington, DC.
[2]. Alok Goyal \& Anil K. Chopra (1989). "Hydrodynamic and Foundation Interaction Effects in Dynamics of Intake Towers: Earthquake Responses", Struct. Engrg., ASCE, 115(4), 1386-1395.

[3]. Em-1110-2-2004-Appendix D-"Refined Procedure for Determination of Added Hydrodynamic Mass". 